\title{
A novel, highly sensitive and specific biomarker for Niemann-Pick type C1 disease
}

\author{
Anne-Katrin Giese ${ }^{1 \dagger}$, Hermann Mascher ${ }^{2 \dagger}$, Ulrike Grittner ${ }^{3}$, Sabrina Eichler ${ }^{4}$, Guido Kramp ${ }^{4}$, Jan Lukas ${ }^{1}$, \\ Danielle te Vruchte ${ }^{5}$, Nada Al Eisa ${ }^{5}$, Mario Cortina-Borja ${ }^{6}$, Forbes D Porter ${ }^{7}$, Frances M Platt ${ }^{5}$ and Arndt Rolfs ${ }^{1,4^{*}}$
}

\begin{abstract}
Background: Lysosomal storage disorders (LSDs), are a heterogeneous group of rare disorders caused by defects in genes encoding for proteins involved in the lysosomal degradation of macromolecules. They occur at a frequency of about 1 in 5,000 live births, though recent neonatal screening suggests a higher incidence. New treatment options for LSDs demand a rapid, early diagnosis of LSDs if maximal clinical benefit is to be achieved.

Methods: Here, we describe a novel, highly specific and sensitive biomarker for Niemann-Pick Type $C$ disease type 1 (NPC1), lyso-sphingomyelin-509. We cross-validate this biomarker with cholestane-3 $\beta, 5 a, 6 \beta$-triol and relative lysosomal volume. The primary cohort for establishment of the biomarker contained 135 NPC1 patients, 66 NPC1 carriers, 241 patients with other LSDs and 46 healthy controls.

Results: With a sensitivity of $100.0 \%$ and specificity of $91.0 \%$ a cut-off of $1.4 \mathrm{ng} / \mathrm{ml}$ was established. Comparison with cholestane-3 $3,5 a, 6 \beta$-triol and relative acidic compartment volume measurements were carried out with a

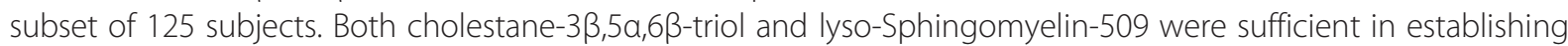
the diagnosis of NPC1 and correlated with disease severity.
\end{abstract}

Conclusion: In summary, we have established a new biomarker for the diagnosis of NPC1, and further studies will be conducted to assess correlation to disease progress and monitoring treatment.

Keywords: Niemann-Pick type C1 disease, NPC1, Biomarker, HPLC-MS/MS

\section{Background}

Lysosomal storage disorders (LSDs) are a group of over 70 different inborn errors of metabolism with a combined incidence around 1 in 5,000 live births [1]. Symptoms depend on tissue distribution and function of the accumulating substrates [2]. Niemann-Pick Type $\mathrm{C}$ disease (NPC) is an autosomal-recessive LSD, that is reported to affect 1:120,000 live births [3], though the relative frequency in at-risk cohorts such as patients with neurologic and psychiatric symptoms, is reported to be as high as $1.2 \%$ [4]. In 95\% of cases NPC is caused by mutations in the NPC1 gene (OMIM: *607623), only

\footnotetext{
* Correspondence: arndt.rolfs@med.uni-rostock.de

${ }^{\dagger}$ Equal contributors

'Albrecht-Kossel-Institute for Neuroregeneration, Medical University of Rostock, Gehlsheimer Str. 20, 18147 Rostock, Germany

${ }^{4}$ Centogene AG, Schillingallee 68, 18055 Rostock, Germany

Full list of author information is available at the end of the article
}

in $5 \%$ of cases have mutations in the NPC2 gene been reported (OMIM: "601015). Currently, Miglustat is the only EMEA-approved drug for the treatment of NPC $[5,6]$, though novel approaches such as cyclodextrin [7] and histone-deacetylase inhibitors [8] are being investigated. Despite clinical evaluation of patients new clinical studies will also require assessment of independent, objective surrogate parameters to evaluate disease progression and response to treatment. In addition to the classical Filipin staining test, which is not always conclusive in patients with NPC [9], two biomarkers for NPC have been reported to date, oxysterols [10] and measurement of intracellular acidic compartment volume (mean equivalent of fluorescence - MEFL/LysoTracker) [11]. While oxysterols facilitate the primary diagnosis of NPC, MEFL is mainly useful in assessing the follow-up and treatment response of patients.

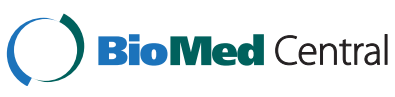


Here, we report the establishment of a novel biomarker for NPC1 and cross-validate our findings with published cholestane-3 $3,5 \alpha, 6 \beta$-triol and MEFL data analyzing the same plasma data [11]. As a first step we screened ten NPC1 patients and 10 age- and gendermatched healthy controls utilizing HPLC and tandem mass spectrometry and carefully assessed differences in mass spectra. This resulted in the detection of lysosphingomyelin-509 (lyso-SM-509) as a potential biomarker for NPC. In the following step, we screened 135 NPC1 patients, 66 NPC1 carriers, 241 patients with other LSDs and 46 healthy controls for lyso-SM-509. The structure is very similar to lyso-sphingomyelin (lyso-SM), the chemical formula being $\left(\mathrm{C}_{24} \mathrm{H}_{49} \mathrm{~N}_{2} \mathrm{O}_{7} \mathrm{P}\right)$, though so far we were not able to elucidate the exact structure of lyso-SM-509. In keeping with this finding, lyso-SM-509 is also highly elevated in patients with NP-A/B, though the disease is easier to diagnose due to availability of enzyme activity assays for sphingomyelinase.

In a second line assessment we compared lyso-SM-509 measurements with those published for cholestane-3 $\beta$, $5 \alpha, 6 \beta$-triol and MEFL. Here, we report on the result of the establishment of lyso-SM-509 as a biomarker and the cross-validation with the previously published biomarkers.

\section{Methods}

\section{Patients and blood samples}

Blood samples were obtained from patients enrolled in the "Biomarker for Niemann-Pick Type C Disease (BioNPC)" trial (ClinicalTrials.gov identifier: NCT01306604) and underwent biochemical analysis or genetic testing for verification of a suspected metabolic disease by the Albrecht-Kossel-Institute for Neuroregeneration (AKos). Additionally, blood plasma samples collected in the scope of the investigation of relative acidic compartment volume in NPC have been analysed for retrospectively for lyso-sphingomyelin-509 levels [11].

Genetic analysis, Oxysterol and LysoTracker measurements Standard analysis of the NPC1 gene was performed according to standard protocols [12]. All measurements for cholestane-3 $\beta, 5 \alpha, 6 \beta$-triol and LysoTracker have been performed previously [11].

\section{Identification of lyso-sphingomyelin-509 as a biomarker for NPC}

Screening for potential biomarkers was carried out using HPLC-MS/MS of blood plasma samples of 10 healthy subjects and 10 NPC1 patients with accurate mass MSsystems (Orbitrap XL) and tandem MS systems. Differences between NPC1 patients and healthy controls were carefully assessed with regards to substances strongly increased only in patients. Molecular weight and structure of such substances were determined carefully. Peaks were identified by accurate mass MS-system and measured by tandem MS/MS systems using already known internal standards as reference. After validating the robustness of lyso-SM-509, measurements in healthy controls, NPC1 carriers, NPC1 patients and in patients with other LSDs ensued (For details see Additional file 1: Figure S1 and S2). Chemical formula determination was done on an LTQ Orbitrap XL, parent ion as well as fragment ions were determined with accurate mass and high resolution leading to the chemical formula described. Purification steps were performed in order to receive larger and purer compound (SPE and liquid-liquid extraction steps-for purification only) to gain higher concentrated compound for HPLC determination and infusion experiments. Sodium aduct ions as well as negatively charged ions of the substance had also been determined and studied more in depth on a $\mathrm{Q} / \mathrm{TOF}$ system. Stress tests of the substance in plasma had been performed, it appears that the substance is stable in plasma over several hours at room temperature, furthermore strongly acidic or alkaline conditions did not show significant degradation. Also several freeze/thaw cycles in human plasma showed stability of the compound.

\section{Method for determination of free lyso-sphingomyelin and lyso-sphingomyelin-509 in plasma}

$50 \mu \mathrm{L}$ of the sample were mixed with $100 \mu \mathrm{L}$ of Internal Standard (lyso-Gb2 (Matreya LLC, USA)) working solution (in $\mathrm{EtOH}$ ) and were then mixed subsequently using a DVX-2500 Multi-tube vortex device at $2500 \mathrm{rpm}$ for about 30 seconds. After centrifugation at $4000 \mathrm{rpm}$ for 2 minutes the clear supernatant was transferred into auto-sampler vials and injected into the HPLC-MS/MS system. Mobile phase used for gradient elution was $50 \mathrm{mM}$ formic acid in water and $50 \mathrm{mM}$ formic acid in acetonitrile/acetone $(1 / 1, \mathrm{v} / \mathrm{v})$. HPLC flow was set at $0.9 \mathrm{~mL} / \mathrm{min}$ on an ACE 3 C8 column $(50 \times 2.1 \mathrm{~mm})$ at $60^{\circ} \mathrm{C}$, the injection volume used was $5 \mu \mathrm{L}$. Retention time for the analytes were approximately 3.2 minutes for lyso-SM and 3.6 minutes for lyso-SM-509, and for the internal standard (lyso-Gb2 (Matreya LLC, USA) approximately 3.2 minutes. Lyso-Gb2 was checked in regards to native concentrations in plasma and was found to be at very low levels only, a sufficient amount was added therefore during sample preparation. The MS/MS system used was an API 4000 using electrospray ionization in MRM mode in positive mode at $500^{\circ} \mathrm{C}$ for determination of free Lyso-Sphingomyelin in plasma. Quadrupole resolution was set at unit /unit, MRM transitions used were $465 \rightarrow 184 \mathrm{~m} / \mathrm{z}$ for the analyte, $509 \rightarrow$ $184 \mathrm{~m} / \mathrm{z}$ for 509 , and $624 \rightarrow 282$ for the internal standard. Calibration was done from $2 \mathrm{ng} / \mathrm{mL}$ to $200 \mathrm{ng} / \mathrm{mL}$ in aqueous/methanolic solution; QC samples were spiked in plasma at levels of 15 and $150 \mathrm{ng} / \mathrm{mL}$. 


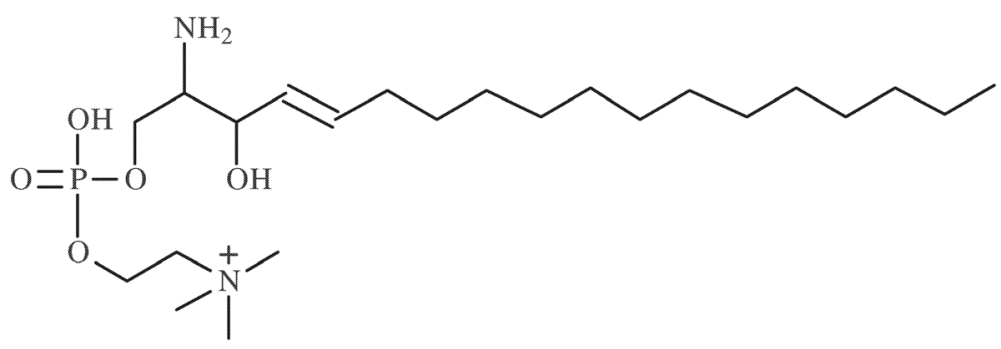

Figure 1 Chemical structure of Lyso-Sphingomyelin-509. This substance was analysed by HPLC-MS/MS with high sensitivity and specificity of diagnosing NPC. The precise structure of lyso-SM-509 has not yet been elucidated.

\section{Statistics}

\section{Statistics for lyso-sphingomyelin-509}

Data were aggregated by taking the first measured value according to genotype (NPC1 patients, NPC1 carriers, NP- A/B patients, P-A/B carriers and healthy controls). Data is given in median and interquartile range where indicated. In order to analyze the diagnostic value of lyso-sphingomyelin-509 a receiver operating characteristic (ROC) curve was employed and the area under the curve (AUC) with 95\%CI was calculated. Descriptive statistics and ROC curve analysis were done using SPSS Release Version 22 (๔ SPSS, Inc., 2013, Chicago, IL, www.spss.com).

\section{For comparison with MEFL and cholestane-3 $3,5 a, 6 \beta$-triol data}

Logistic regression models were fitted to compare proportions, Wilcoxon-Mann-Whitney and Kruskal-Wallis tests were used to compare medians, and ANOVA was used to compare means where appropriate. Linear regression models were fitted with maximum likelihood. A locally adaptive super smoother was used to extract the data structure without a regression model [13]. These calculations were performed in the $R$ language and environment for statistical computing (version 2.14.2; http://www.R-project.org). Graphs with error bars represent mean \pm SD. A $p$-value smaller than 0.05 was considered significant.

\section{Study approval}

The protocol of the BioNPC trial has been approved by the Research Ethics Committee of the University Rostock (ClinicalTrials.gov identifier: NCT01306604). Patients undergoing therapy were treated according to standard protocols. Written informed consent was obtained from all participants or their legal guardians.

\section{Results and discussion}

Establishment of lyso-sphingomyelin-509 as a biomarker for NPC and characterization of the study cohort

The recently emerging novel treatment approaches for treating NPC including cyclodextrin [7], HSP70 and HDAC inhibitors [8] strengthen the need for early diagnosis and monitoring of disease progression. Biomarkers for LSDs have been investigated for some time to facilitate diagnosis and monitoring response to therapy. In, 1989 Rosengren and colleagues investigated the glycosphingolipid pattern in late infantile metachromatic

Table 1 Characteristics of investigated populations

\begin{tabular}{|c|c|c|c|c|c|}
\hline Cohort & Individuals (n) & Measurements $(n)$ & $\begin{array}{l}\text { Age (first value) } \\
\text { (median, IQR, number of cases) }\end{array}$ & $\begin{array}{l}\text { Males ( } n \text { ) } \\
\text { Age (median, IQR) }\end{array}$ & $\begin{array}{l}\text { Females ( } n) \\
\text { Age (median, IQR) }\end{array}$ \\
\hline \multirow[t]{2}{*}{ NPC1 patients } & 110 & 241 & $13(4-20)$ & 33 & 33 \\
\hline & & & $(n=67)$ & $13(4-19)$ & $12(5-23)$ \\
\hline \multirow[t]{2}{*}{ NPC1 carriers } & 63 & 94 & $46(25-54)$ & 8 & 12 \\
\hline & & & $(n=20)$ & $48(27-56)$ & $45(14-53)$ \\
\hline \multirow[t]{2}{*}{ NP-A/B patients } & 21 & 21 & $9(1-15)$ & 10 & 4 \\
\hline & & & $(n=14)$ & $7(1-15)$ & $11(3-47)$ \\
\hline \multirow[t]{2}{*}{ NP-A/B carriers } & 5 & 5 & 7 (min-max: 2-35) & 1 & 2 \\
\hline & & & $(n=3)$ & $7 \mathrm{yrs}$ & Med:: 19 \\
\hline \multirow[t]{2}{*}{ Healthy controls } & 43 & 47 & $41(20-50)$ & 3 & 9 \\
\hline & & & $(n=12)$ & 51 (min-max: 4-71)) & $38(21-46)$ \\
\hline
\end{tabular}



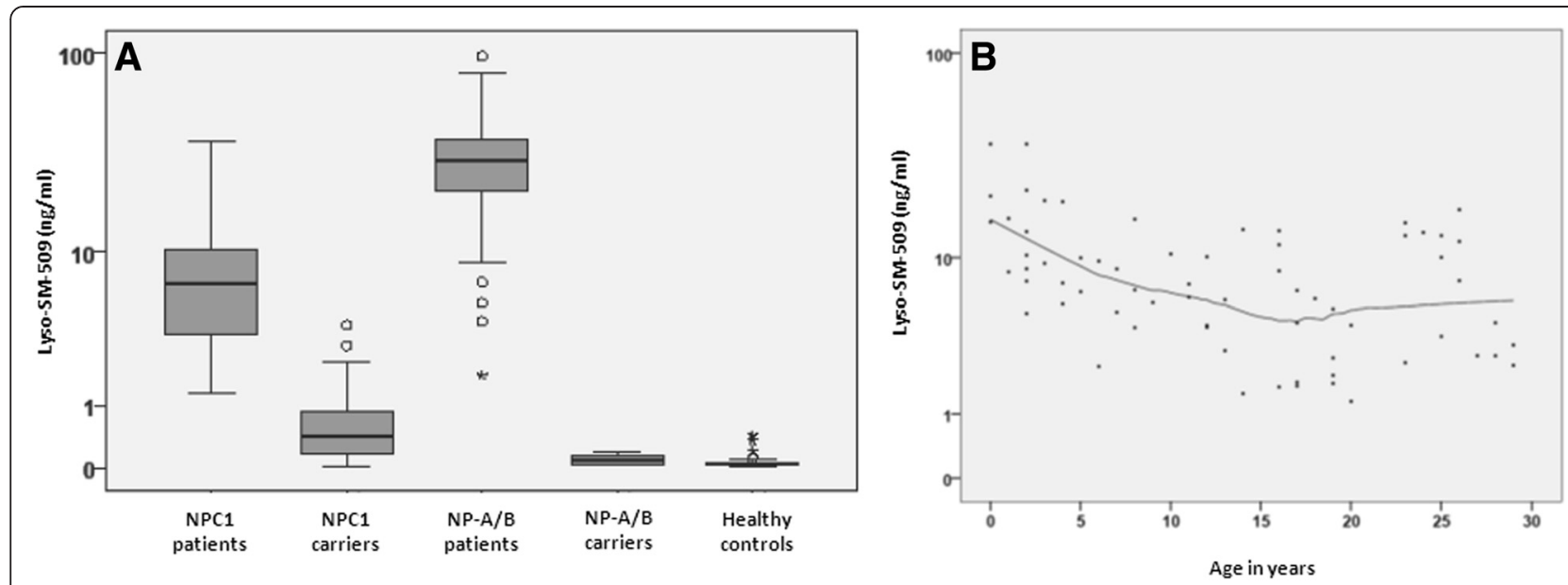

Figure 2 Levels of lyso-SM-509 in patients with NPC1 $(n=110), N P-A / B(n=21)$, as well as healthy controls $(n=43)$ and NPC1 carriers $(n=63)$ and NP-A/B carriers $(n=5)$. (A) Lyso-SM-509 allows distinguishing between healthy controls and patients with NPC1. Notably, the biomarker levels are for NP-A/B patients were 3.55-times higher (29.4 ng/ml (IQR: 14.8-40.0). Notably, only in two cases lyso-SM-509 levels above $2.5 \mathrm{ng} / \mathrm{ml} \mathrm{can}$ be detected in a carrier of a NPC1 mutation and in none of the healthy controls. (B) Levels of lyso-SM-509 in NPC1 by age. Note that Lyso-SM-509 is higher in younger NPC1 patients $(\rho=-0.519, p<0.001)$, reflecting the severity of early onset disease as shown by Te Vruchte and colleagues (11).

leukodystrophy (MLD) [14]. Lysosulfatide had been identified in MLD and normal brain tissue subsequently, the authors arguing for a de novo synthesis of lysoglycosphingolipids from sphingosine and arrive at the conclusion that they do not play a role in disease pathology [14].

Since then the perception of lysoglycosphingolipids has changed dramatically. For example several lysosphingolipid biomarkers having been established recently, e.g. lyso-Globotriaosylceramide (lyso-Gb3) in Fabry disease [15], Glucosylsphingosine (lyso-GL-1) in Gaucher disease [16], Galactosylsphingosine (Psychosine) in Krabbe disease $[17,18]$ and lyso-sphingomyelin in Niemann-Pick B patients [19].

Here, we investigated a potential biomarker for NPC1, lyso-SM-509. For the establishment of a biomarker for NPC1 the plasma samples of 10 NPC1 patients and 10 healthy controls were analyzed by tandem mass spectrometry as described in the Material and Methods section. After initial testing, lyso-SM-509 (Figure 1, for additional information see Supplement) was determined as an interesting target for the primary diagnosis of NPC1. Though we have established $\mathrm{C}_{24} \mathrm{H}_{49} \mathrm{~N}_{2} \mathrm{O}_{7} \mathrm{P}$ as the molecular formula for lyso-SM-509 (lyso-SM with the addition of

Table 2 Medians and interquartile ranges for lyso-SM-509 in $\mathrm{ng} / \mathrm{ml}$ for all investigated sub-cohorts

\begin{tabular}{lrl}
\hline & \multicolumn{1}{l}{} & Lyso-SM-509 in $\mathbf{n g} / \mathbf{m l}$ \\
\hline NPC1 patients & 110 & $6.7(3.4-10.3)$ \\
NPC1 carriers & 63 & $0.4(0.2-0.9)$ \\
NP- A/B patients & 21 & $29.4(14.8-40.0)$ \\
NP- A/B carrier & 5 & $0.09(0.04-0.16)$ \\
Healthy controls & 43 & $0.04(0.03-0.06)$ \\
\hline
\end{tabular}

carbon dioxide, the compound was dubbed for the mass weight examined, Figure 1), the exact structure is still subject of investigation. Current results suggest that the carbon dioxide is most likely located at the amino group of the lyso-SM (data not shown). A calculation of peak area ratio to internal standard was used for lyso-SM-509. To date, the mechanism by which lysoglycosphingolipids arise has not been elucidated, the most prominent hypothesis, also for lyso-Gb3 and lyso-GL1, being the deacylation of the corresponding glycosphingolipid [15,16,20].

Overall, 242 subjects with originally 408 measurement points were analyzed, the main cohorts were (1) NPC1 patients $(n=110$, median age 13 years, (2) NPC1 carriers ( $n=63$, median age: 46 , (3) NP-A/B patients $(n=21$, median age: 9 , (4) NP-A/B carriers $(n=5$, median age: 7$)$, and (5) healthy controls $(n=43$, median age: 41 (for details see Table 1 ). For NPC1 patients a median lyso-SM509 value of $6.7 \mathrm{ng} / \mathrm{ml}$ was measured, NP-A/B patients displayed about 4-times higher values $(29.4 \mathrm{ng} / \mathrm{ml})$, while neither NPC1 carriers, NP-A/B carriers, nor healthy controls displayed elevated values (for details see Figure 2A, B and Table 2). Of note, in two instances NPC1 carriers

Table 3 Sensitivity, specificity and accuracy of lyso-SM-509 in NPC1 patients

\begin{tabular}{ll}
\hline & Lyso-SM-509 \\
\hline NPC1 patients $(\boldsymbol{n})$ & $110(221)$ \\
Cut point/sensitivity range $(\mathrm{ng} / \mathrm{ml})$ & 1.4 \\
Sensitivity & $100.0 \%$ \\
Specificity & $91.0 \%$ \\
Accuracy & $95.5 \%$ \\
AUC and $95 \% \mathrm{Cl}$ in ROC Analysis & $0.99(0.98-1.00)$ \\
\hline
\end{tabular}



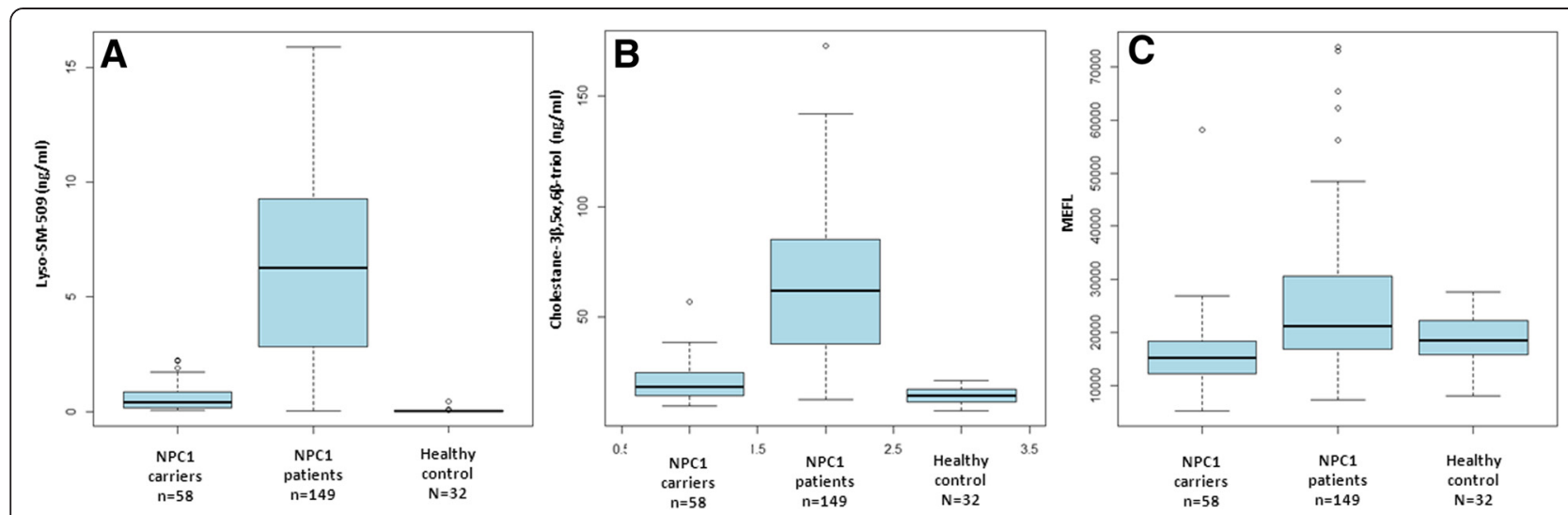

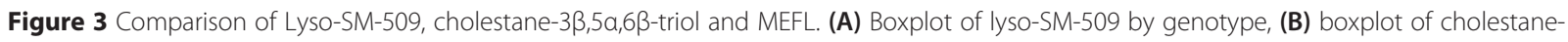

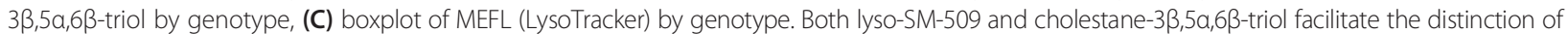
NPC patients from healthy controls.

displayed measurements above $2.5 \mathrm{ng} / \mathrm{ml}$. Each were parents of genetically confirmed NPC1 patients and both underwent genotyping confirming their carrier status. In summary, a cut-point of $1.4 \mathrm{ng} / \mathrm{ml}$ for lyso-SM-509 was determined to distinguish NPC1 patients from NP-A/B patients, NPC1 carriers, NP-A/B carriers and healthy controls (sensitivity: 100.0\%, specificity: $91.0 \%$, ROC-curve analysis: Area under the curve (AUC): 0.99, 95\% CI: 0.981.00) (Table 3). We conclude that lyso-SM-509 is useful in determining the primary diagnosis of NPC1.

\section{Relationship to MEFL and cholestane $3 \beta, 5 \alpha, 6 \beta$-triol}

For NPC1, two other biomarkers have already been established - non-enzymatically generated Oxysterols and the LysoTracker (by assessing MEFL as a measure of relative lysosomal volume) [10,11]. In the second part of our paper, the newly established lyso-SM-509 has been compared to both in the same plasma samples. Even though, two different biomarkers have been established and can be utilized for different aspects of the disease (Oxysterols for primary diagnosis by measurement of either Cholestane-

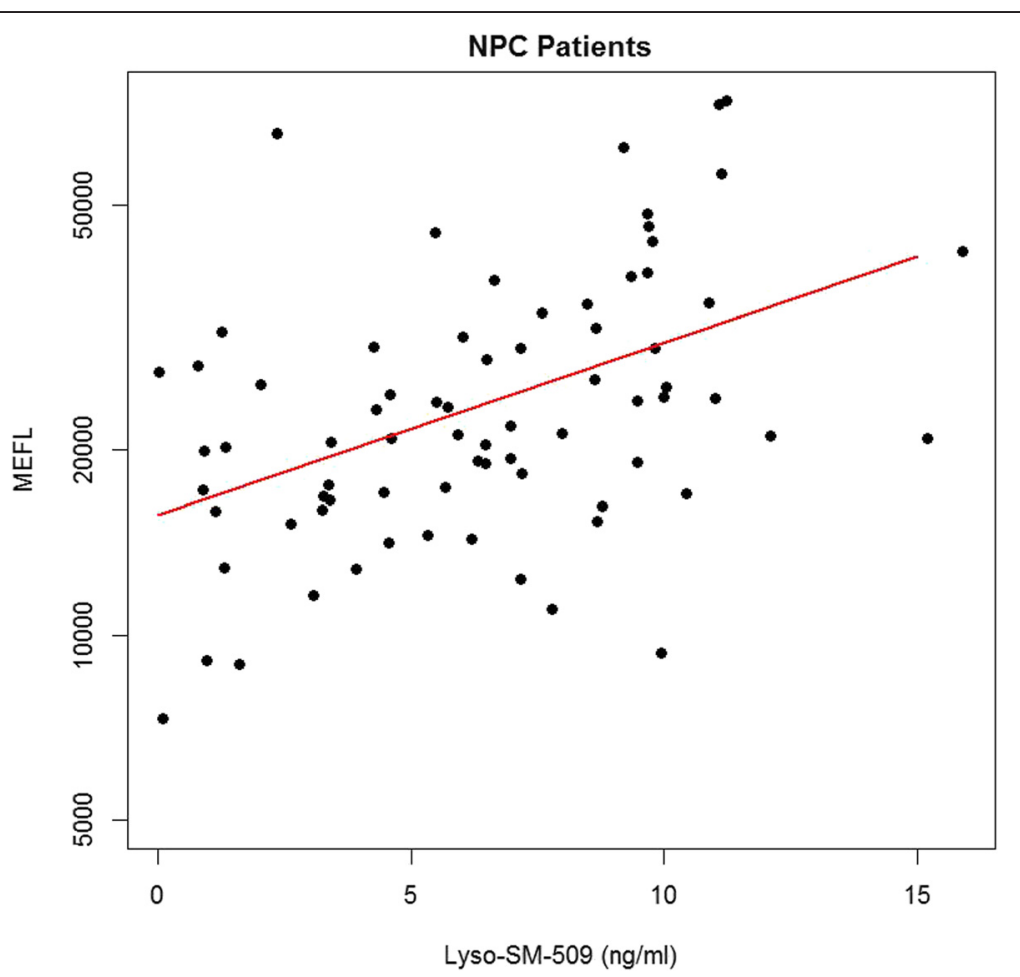

Figure 4 Comparison of MEFL (LysoTracker) (log scale) and lyso-SM-509 (linear scale). Levels of lyso-SM-509 significantly correlate with MEFL in NPC1 patients $(\rho=0.432, p<0.001)$. The Red line is a linear model. 


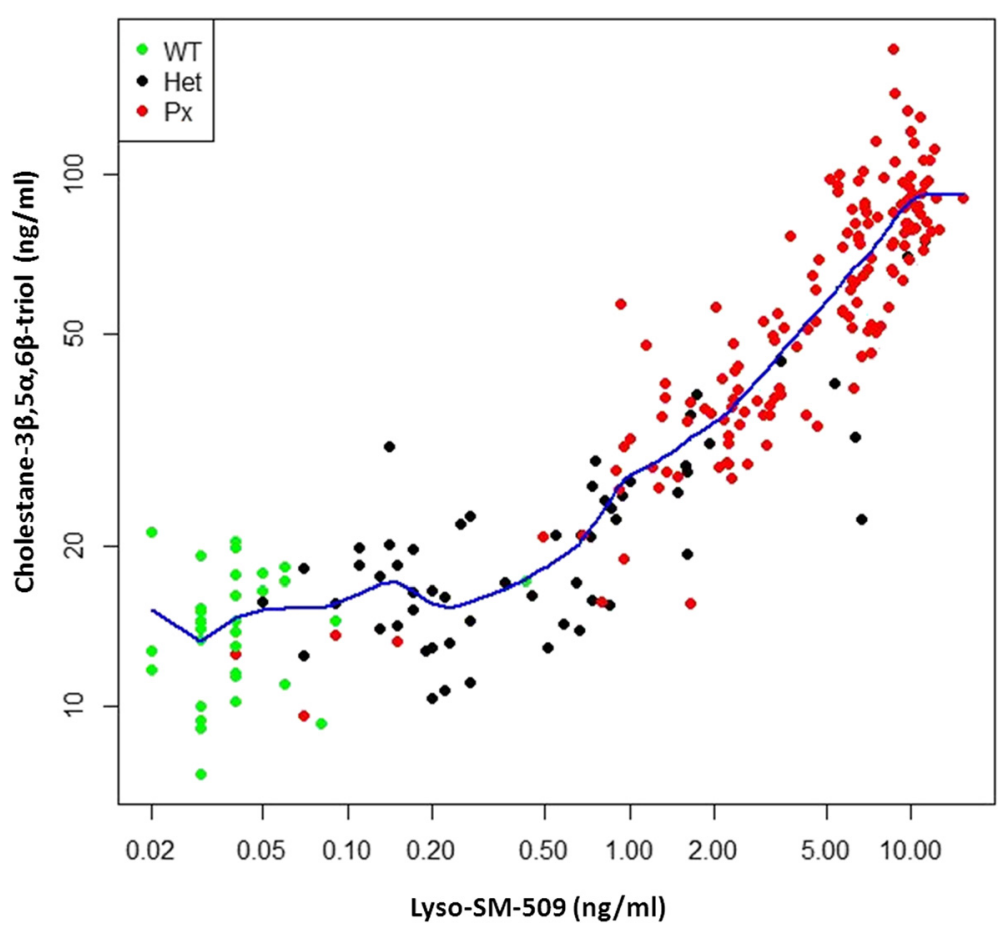

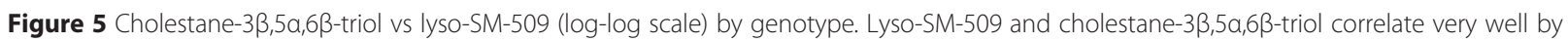
genotype (Healthy controls, NPC1 carriers and NPC1 patients; $\rho=0.675, p<0.001$ ); blue line is a super smoother.

$3 \beta, 5 \alpha, 6 \beta$-triol or 7-ketocholesterol, which allows for discrimination between controls and NPC1 subjects [10] vs. MEFL for monitoring of disease progression and treatment response, which has been assessed in a 5-year prospective study [11]), an ideal biomarker for the primary diagnosis, monitoring of disease progression and treatment response has not yet been established. Lysosphingomyelin-509 as a biomarker for NPC adds discriminatory power to identify and will further facilitate early disease diagnosis. In fact, many diseases - even common diseases such as myocardial infarction - involve several biomarkers being used for primary diagnosis and follow-up. Therefore, our findings significantly contribute to the current literature on diagnosing NPC by adding a new biomarker.

In order to investigate the relationship of lysosphingomyelin-509 to MEFL and cholestane-3 $\beta, 5 \alpha, 6 \beta-$ triol (Oxysterols), we measured its values in 240 samples

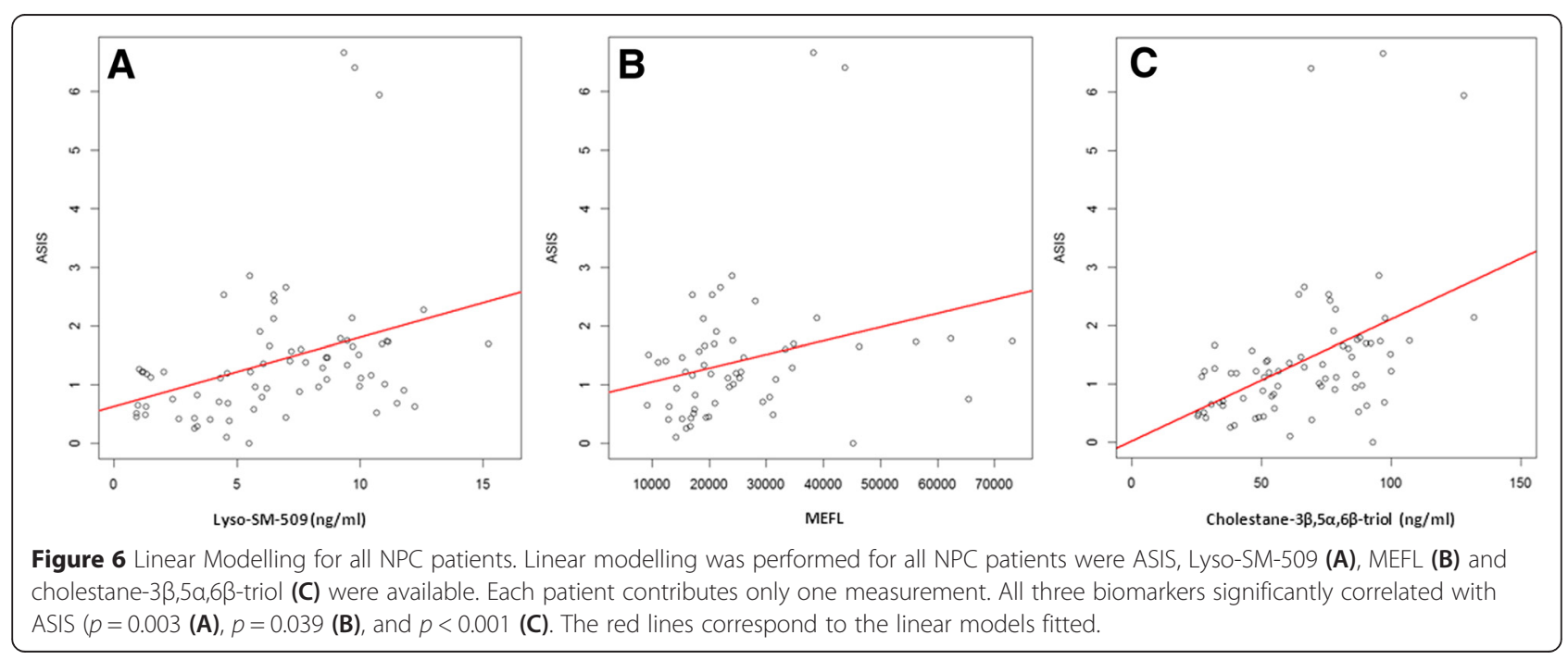


from 125 subjects (NPC patients, NPC heterozygotes and healthy controls) that were previously collected and analyzed by FM Platt and colleagues in the scope of a 5year international prospective study investigating lysosomal volume in B cells from NPC patients [11]. Data for this subcohort of samples are summarized in Figure 3A-C. Levels of lyso-SM-509 correlate significantly with MEFL in NPC1 patients; $\rho$ denotes Spearman's correlation coefficient (Figure $4, \rho=0.432 p<0.001$ ). In addition, lyso-SM-509 levels correlate very well with cholestane- $3 \beta, 5 \alpha, 6 \beta$-triol $(\rho=0.921, p<0.001)$ (Figure 5) that had been measured in the same samples and the values previously published [10], samples overlapping with controls (lyso-SM-509< $2.5 \mathrm{ng} / \mathrm{ml}$ ) were excluded for the analysis. Taken together, lyso-SM-509 correlated well with both cholestane- $3 \beta, 5 \alpha$, $6 \beta$-triol and MEFL, though lyso-SM-509 and cholestane$3 \beta, 5 \alpha, 6 \beta$-triol were very highly correlated, suggesting they may be generated at least in part by the same process. In line with this, the LysoTracker assay was found to be semi-independent of both biochemical markers and combining the MEFL with either one adding discriminatory power to the analysis.

\section{Correlation of biomarkers with severity scores}

A randomly selected subgroup of 73 samples with measurements from 36 NPC1 patients for cholestane-3 $\beta$, $5 \alpha, 6 \beta$-triol and lyso-SM-509 (median age: 12.76 (IQR: 9.83-17.28)) and MEFL with 57 measurements in 30 patients (median age: 13.00 (IQR: $10.38-18.49)$ ) were analyzed for correlations with the total severity score of the patients (excluding hearing). In agreement with previous studies on the cholestane-3 $\beta, 5 \alpha, 6 \beta$-triol and MEFL there were no significant correlations with any of these biomarkers (data not shown) [10,11]. We also found no significant correlation between lyso-SM-509 and total severity score $(\rho=0.031, p=0.794)$. However, as NPC1 is a progressive neurodegenerative disorder, and patients continue to progress over a number of years the annual severity increment score (ASIS) [11] was calculated by dividing the total severity score by age to determine relative rate of disease progression. As in the original NPC cohort study carried out by Frances Platt and colleagues [11], hearing was excluded since it was not measured in all centers. Lyso-SM-509 was significantly correlated to ASIS (slope $p=0.003$ ), as were cholestane- $3 \beta, 5 \alpha, 6 \beta$-triol (slope $p<0.001$ ) and MEFL (slope $p=0.039$ ) (Figure 6). Analysis of the effect of therapy (miglustat treated vs untreated patients) was underpowered due to low numbers of untreated patients $(n-=21)$ so a larger study will be needed to address this.

\section{Conclusion}

In summary, we have established a novel, sensitive and specific biomarker for the primary diagnosis of NPC1.
The major advantage of lyso-SM-509 is that it is quick and easy to measure and standardize. As a consequence, lyso-SM-509 is definitely a useful biomarker for simple diagnostic in blood plasma, future investigations will analyze the sensitivity and specificity of lyso-SM-509 on dried blood spot samples, which would further simplify the diagnostic process.

\section{Additional file}

\begin{abstract}
Additional file 1: Figure S1. Chromatogram of lyso-SM-509 (top chromatogram) and the internal standard (bottom) in human control plasma. There is almost no lyso-SM-509 detectable in the control sample. Figure S2. Chromatogram of lyso-SM-509 (top chromatogram) and internal standard (bottom) in NPC1 patient sample at a high concentration.
\end{abstract}

\section{Competing interests}

The authors declare that they have no competing interests.

\section{Authors' contributions}

AKG and AR conceived the study and carried out the design and coordination of the study. AKG, HM and AR drafted the manuscript. HM performed the biochemical assays, evaluated the primary data and drafted the manuscript. UG performed the statistical analysis and helped to draft the manuscript. SE carried out the genetic analysis. GK carried out biochemical assays. $J$ participated in the evaluation of the data and helped to draft the manuscript. DtV, NAE, MCB and FMP participated in the design of the study, statistical analysis and helped to draft the manuscript. FDP provided samples and coded subject demographic and severity score data. All authors read and approved the final manuscript.

\section{Acknowledgments}

The authors would like to thank all patients and clinical partners who have contributed to the recruitment of the patients. Special thanks are extended to Susanne Zielke, Sabine Rösner, Frances König, Vivian Kersten and Lea Maciolek for important support in the organization of the clinical study. F.M.P. is a Royal Society Wolfson Research Merit Award holder. The University College London Institute of Child Health receives a portion of its funding from the UK Department of Health's NIH Research Biomedical Research Funding Centres funding scheme. DtV is supported by Action Medical Research and an unrestricted grant from Actelion. FDP is supported by the intramural research program of the Eunice Kennedy Shriver National Institute of Child Health and Human Development, NIH.

\section{Author details}

${ }^{1}$ Albrecht-Kossel-Institute for Neuroregeneration, Medical University of Rostock, Gehlsheimer Str. 20, 18147 Rostock, Germany. ${ }^{2}$ PharmAnalyt Labor GmbH, Ferdinand-Pichler Gasse 2, 2500 Baden, Austria. ${ }^{3}$ Department for Biostatistics and Clinical Epidemiology, Charité-University Medical Centre, Berlin, Germany. ${ }^{4}$ Centogene AG, Schillingallee 68, 18055 Rostock, Germany. ${ }^{5}$ Department of Pharmacology, University of Oxford, Mansfield Road, Oxford OX1 3QT, UK. ${ }^{6}$ Population, Policy and Practice Programme, Institute of Child Health, University College London, 30 Guilford Street, London WC1N 1EH, UK. ${ }^{7}$ Eunice Kennedy Shriver National Institute of Child Health and Development, National Institutes of Health, 10 Center Drive, Bethesda, MD 20892, USA

Received: 9 January 2015 Accepted: 24 April 2015

Published online: 17 June 2015

References

1. Fuller M, Meikle PJ, Hopwood JJ. Epidemiology of lysosomal storage diseases: an overview. Oxford: Oxford PharmaGenesis; 2006. p. 2006. Chapter 2.

2. Wang RY, Bodamer OA, Watson MS, Wilcox WR, ACMG Work Group on Diagnostic Confirmation of Lysosomal Storage Diseases. Lysosomal storage 
diseases: diagnostic confirmation and management of presymptomatic individuals. Genet Med. 2011;13(5):457-84.

3. Mengel E, Klünemann HH, Lourenço CM, Hendriksz CJ, Sedel F, Walterfang $M$, et al. Niemann-Pick disease type $C$ symptomatology: an expert-based clinical description. Orphanet J Rare Dis. 2013;8:166.

4. Bauer $P$, Balding DJ, Klünemann $H H$, Linden DE, Ory DS, Pineda $M$, et al. Genetic screening for Niemann-Pick disease type $C$ in adults with neurological and psychiatric symptoms: findings from the ZOOM study. Hum Mol Genet. 2013;22(21):4349-56.

5. Patterson MC, Vecchio D, Prady H, Abel L, Wraith JE. Miglustat for treatment of Niemann-Pick C disease: a randomised controlled study. Lancet Neurol. 2007;6(9):765-72.

6. Patterson MC, Hendriksz CJ, Walterfang M, Sedel F, Vanier MT, Wijburg F, et al. Recommendations for the diagnosis and management of NiemannPick disease type C: an update. Mol Genet Metab. 2012;106(3):330-44.

7. Ottinger EA, Kao ML, Carrillo-Carrasco N, Yanjanin N, Shankar RK, Janssen M, et al. Collaborative development of 2-hydroxypropyl- $\beta$-cyclodextrin for the treatment of Niemann-Pick type C1 disease. Curr Top Med Chem. 2014;14(3):330-9.

8. Helquist P, Maxfield FR, Wiech NL, Wiest O. Treatment of Niemann-pick type $C$ disease by histone deacetylase inhibitors. Neurotherapeutics. 2013;10(4):688-97.

9. Vanier MT, Rodriguez-Lafrasse C, Rousson R, Gazzah N, Juge MC, Pentchev $P G$, et al. Type C Niemann-Pick disease: spectrum of phenotypic variation in disruption of intracellular LDL-derived cholesterol processing. Biochim Biophys Acta. 1991;1096(4):328-37.

10. Jiang X, Sidhu R, Porter FD, Yanjanin NM, Speak AO, te Vruchte DT, et al. A sensitive and specific LC-MS/MS method for rapid diagnosis of NiemannPick C1 disease from human plasma. J Lipid Res. 2011;52(7):1435-45.

11. te Vruchte D, Speak AO, Wallom KL, Al Eisa N, Smith DA, Hendriksz CJ, et al. Relative acidic compartment volume as a lysosomal storage disorderassociated biomarker. J Clin Invest. 2014;124(3):1320-8.

12. Bauer P, Knoblich R, Bauer C, Finckh U, Hufen A, Kropp J, et al. NPC1: Complete genomic sequence, mutation analysis, and characterization of haplotypes. Hum Mutat. 2002;19(1):30-8.

13. Friedman JH. A variable span scatterplot smoother. Laboratory for Computational Statistics. In: Stanford University Technical Report No. 5. 1984.

14. Rosengren B, Fredman P, Månsson JE, Svennerholm L. Lysosulfatide (galactosylsphingosine-3-O-sulfate) from metachromatic leukodystrophy and normal human brain. J Neurochem. 1989;52:1035-41.

15. Aerts JM, Groener JE, Kuiper S, Donker-Koopman WE, Strijland A, Ottenhoff $\mathrm{R}$, et al. Elevated globotriaosylsphingosine is a hallmark of Fabry disease. Proc Natl Acad Sci U S A. 2008;105(8):2812-7.

16. Rolfs A, Giese AK, Grittner U, Mascher D, Elstein D, Zimran A, et al. Glucosylsphingosine is a highly sensitive and specific biomarker for primary diagnostic and follow-up monitoring in Gaucher disease in a non-Jewish, Caucasian cohort of Gaucher disease patients. PLoS One. 2013;8(11):e79732.

17. Galbiati F, Givogri Ml, Cantuti L, Rosas AL, Cao H, van Breemen R, et al. Combined hematopoietic and lentiviral gene-transfer therapies in newborn Twitcher mice reveal contemporaneous neurodegeneration and demyelination in Krabbe disease. J Neurosci Res. 2009;87(8):1748-59.

18. Chuang WL, Pacheco J, Zhang XK, Martin MM, Biski CK, Keutzer JM, et al. Determination of psychosine concentration in dried blood spots from newborns that were identified via newborn screening to be at risk for Krabbe disease. Clin Chim Acta. 2013;419:73-6.

19. Chuang WL, Pacheco J, Cooper S, McGovern MM, Cox GF, Keutzer J, et al. Lyso-sphingomyelin is elevated in dried blood spots of Niemann-Pick B patients. Mol Genet Metab. 2014;111(2):209-11.

20. Dekker N, van Dussen L, Hollak CE, Overkleeft H, Scheij S, Ghauharali K, et al. Elevated plasma glucosylsphingosine in Gaucher disease: relation to phenotype, storage cell markers, and therapeutic response. Blood. 2011;118(16):e118-27.

\section{Submit your next manuscript to BioMed Central and take full advantage of:}

- Convenient online submission

- Thorough peer review

- No space constraints or color figure charges

- Immediate publication on acceptance

- Inclusion in PubMed, CAS, Scopus and Google Scholar

- Research which is freely available for redistribution 